archives-ouvertes

\title{
Both dog and human faces are explored abnormally by young children with autism spectrum disorders
}

Quentin Guillon, Nouchine Hadjikhani, Sophie Baduel, Jeanne Kruck, Mado Arnaud, Bernadette Rogé

\section{To cite this version:}

Quentin Guillon, Nouchine Hadjikhani, Sophie Baduel, Jeanne Kruck, Mado Arnaud, et al.. Both dog and human faces are explored abnormally by young children with autism spectrum disorders. NeuroReport, Lippincott, Williams \& Wilkins, 2014, 10.1097/WNR.0000000000000257 . hal-02476868

\section{HAL Id: hal-02476868 \\ https: / hal.archives-ouvertes.fr/hal-02476868}

Submitted on 13 Feb 2020

HAL is a multi-disciplinary open access archive for the deposit and dissemination of scientific research documents, whether they are published or not. The documents may come from teaching and research institutions in France or abroad, or from public or private research centers.
L'archive ouverte pluridisciplinaire HAL, est destinée au dépôt et à la diffusion de documents scientifiques de niveau recherche, publiés ou non, émanant des établissements d'enseignement et de recherche français ou étrangers, des laboratoires publics ou privés. 


\section{Both dog and human faces are explored abnormally by young children with autism spectrum disorders Quentin Guillon ${ }^{\mathrm{a}}$, Nouchine Hadjikhani ${ }^{\mathrm{b}, \mathrm{c}}$, Sophie Baduel ${ }^{\mathrm{a}}$, Jeanne Kruck
Mado Arnaud $^{\mathrm{a}}$,}

\begin{abstract}
When looking at faces, typical individuals tend to have a right hemispheric bias manifested by a tendency to look first toward the left visual hemifield. Here, we tested for the presence of this bias in young children with autism spectrum disorders (ASD) for both human and dog faces. We show that children with ASD do not show a left visual hemifield (right hemispheric) bias for human faces. In addition, we show that this effect extends to faces of dogs, suggesting that the absence of bias is not specific to human faces, but applies to all faces with the first-order configuration, pointing to an anomaly at an early stage of visual analysis of faces. The lack of right hemispheric dominance for face processing may reflect a more general disorder of cerebral specialization of social functions
\end{abstract}

\section{Introduction}

Eye-tracking studies of face perception have showed that typical individuals tend to explore the left side of a face (from the viewer's perspective) first and for a longer time if the face is presented at the central vision [1-8]. This left visual hemifield gaze bias has been found in typically developing children as young as 6 months $[3,6,8]$, and is independent of the task [4], the emotional valence [7], the race [6], and even the species of the face - human or animal [3,7]. The current hypothesis links this bias to the right hemispheric dominance for face processing [4]. According to this view, the left side of a face would be more salient than the right side because it is initially projected to the right hemisphere $[1,9]$.

Autism spectrum disorders (ASD) are conditions that affect more than $1 \%$ of the population [10] and in which social communication is affected. In particular, difficulties are noted in the domain of face perception. Despite these difficulties, only a few studies have investigated hemispheric lateralization for face processing in ASD. This is all the more surprising, given the atypicalities observed in this area, both at the behavioral level and at the electrophysiological level [11-13]. EEG studies have reported a selective processing delay for faces in the right hemisphere and a reduced right lateralization effect for faces compared with controls [11,12]. At the behavioral level, Dundas et al. [14] have reported a lack of a left visual hemifield bias for the proportion of looking time during face perception in high-functioning adults.

The aim of the present study was to investigate the presence of a gaze bias for the left visual hemifield in in ASD. NeuroReport 00:000-000 (c) 2014 Wolters Kluwer Health | Lippincott Williams \& Wilkins.

NeuroReport 2014, 00:000-000

Keywords: autism spectrum disorders, eye-tracking, face perception, hemispheric lateralization

aURI Octogone-CERPP, University of Toulouse, France, b Harvard Medical School/MGH/MIT, Martinos Center for Biomedical Imaging, Charlestown, Massachusetts, USA and ${ }^{\circ}$ Gillberg Neuropsychiatry Center, Gothenburg University, Sweden

Correspondence to Quentin Guillon, PhD, URI Octogone-CERPP, University of Toulouse, 5 allée Antonio Machado, 31058 Toulouse Cedex9, France Tel: +335615024 25; fax: + 335615049 18;

e-mail: quentin.guillon@univ-tlse2.fr

Received 26 June 2014 accepted 4 August 2014

response to the presentation of human and canine faces in young children with ASD. Unlike Dundas et al. [14], we specifically focused on the direction of the first fixation as it is an automatic response considered to reflect directly the right hemispheric dominance for face processing [1]. We tested the hypothesis that contrary to neurotypical children, young children with ASD would not show a left visual hemifield bias for the direction of the first fixation for faces.

\section{Methods}

Participants

All parents provided their free and informed consent to participate in the study in accordance with the Declaration of Helsinki (6th revision), and all procedures were approved by the local ethical committee.

A total of 47 children (20 children with ASD), aged 24-60 months, participated in this study. One child with ASD and six control children had to be excluded because of attention problems during the data acquisition or visual disorders. The data from 19 children with ASD and 22 typically developing (TD) children were included in the analyses.

The diagnosis of ASD was established with the ADOS-G [15] and the ADI-R [16]. The diagnosis was confirmed by at least one clinical psychologist trained and certified in making a diagnosis of ASD on the basis of these tools and with experience in diagnosing young children (B.R. or J.K.).

Exclusion criteria for typically developing children were first-degree relation to a child with neurodevelopmental 
disorder, visual impairment, neurological disorder, genetic disorder, and developmental delay at the time of inclusion.

Mullen Scales of Early Learning were used to assess nonverbal and verbal developmental levels in all children [17].

No difference was observed between groups for chronological age [in months: mean $_{\mathrm{ASD}}=39.6, \mathrm{SD}=10.5$; mean $\left._{\mathrm{TD}}=43.1, \mathrm{SD}=14.6 ; t(39)=-0.854 ; P=0.398\right]$ and the sex ratio [boy/girl: ASD: $15 / 4$; TD: $13 / 9, \chi^{2}(1$, $N=41)=1.86, P=0.173$ ]. However, the nonverbal and verbal mental ages were significantly lower for the ASD group [in months: NVMA: mean $_{\mathrm{ASD}}=32.3, \mathrm{SD}=11.7$; mean $_{\mathrm{TD}}=45.1, \quad \mathrm{SD}=14.9 ; \quad t(39)=-3.02 ; \quad P=0.005 ;$ VMA: $\operatorname{mean}_{\mathrm{ASD}}=27.4, \quad \mathrm{SD}=13.6 ; \quad \operatorname{mean}_{\mathrm{TD}}=44.0$, $\mathrm{SD}=14.1 ; t(39)=-3.85 ; P<0.001]$.

\section{Stimuli}

The stimuli were used previously by Racca et al. [7]. They consisted of 28 grayscale photographs, including 12 photographs of faces selected from the Ekman and Friesen database [18]. These were further divided into four faces (two women) with a negative facial expression (anger), four faces (two women) with a neutral facial expression, and four faces (two women) with a positive facial expression (happy). Twelve photographs were canine faces. As for the human faces, four canine faces had negatively valenced expression, four faces were neutral, and four faces were positively valenced. An additional four photographs of symmetrical objects with respect to a vertical axis (flower, tree, house, and shoe) completed the set of stimuli (Fig. 1).

All photographs were $19^{\circ} \times 20^{\circ}$ at a distance of $60 \mathrm{~cm}$. All had a uniform gray background so that the left and the right side of each stimulus did not differ in terms of luminance $\left(\mathrm{cd} / \mathrm{m}^{2}\right)$ and contrast (percentage of 'gray' pixels) to control for an effect of the low-level properties of the stimulus on eye movements. The stimuli were presented using E-prime 2.0 software (Psychology Software Tools Inc., Pittsburgh, Pennsylvania, USA) on a 17 -inch screen $(1024 \times 768$ resolution $)$, integrated to a Tobii T120 eye-tracking system (Tobii Inc., Stockholm, Sweden). The pupil and corneal reflections were recorded at a sampling rate of $60 \mathrm{~Hz}$.

\section{Procedure}

The recordings were made in a room where light levels were maintained constant for all the participants. All potential distractors were removed, and children sat in a car seat adapted and fixed to a chair with an adjustable height at a distance of about $60 \mathrm{~cm}$ from the screen. If they so desired, parents could stay with their children, provided that they remained behind the seat and did not talk to the child during the experiment.
A five-point calibration was performed. The calibration point was a video $\left(1.5^{\circ} \times 1.5^{\circ}\right)$ representing a bouncing ball with a sound. If less than four points per eye were properly calibrated, a new calibration was performed until it was satisfactory (i.e. $\geq 4$ focus properly calibrated for each eye).

Each trial began with a central fixation point $\left(1.5^{\circ} \times 1.5^{\circ}\right)$ representing a spinning top on a gray background. For the stimuli to appear, the child had to look at the spinning top for 300 consecutive milliseconds within a time window of $5 \mathrm{~s}$ after its onset. This procedure ensured that all children looked at the same place when the stimuli appeared. In case the participant did not look at the spinning top, the stimuli were displayed after $5 \mathrm{~s}$ had elapsed, but the trial was considered invalid and not included in the analysis. The stimuli were presented for $3500 \mathrm{~ms}$ in a random order, followed by a gray screen with a sound varying from 500 to $800 \mathrm{~ms}$ to retain the attention of the child.

\section{Gaze data analysis}

All data analyses were carried out with Matlab (R2011a; MathWorks, Natick, Massachusetts, USA) using in-house scripts. Fixations were identified using algorithms from [19]. A velocity threshold of $35 \%$ and a temporal threshold of $100 \mathrm{~ms}$ were used to identify fixations.

First fixation was considered to be in toward the left or the right visual hemifield when their amplitude was equal to or larger than $1^{\circ}$ relative to the central fixation in that direction. Fixations that fell within $1^{\circ}$ of the center were considered non-lateralized fixations.

For each participant and each face category, a laterality index for the direction of the first fixation was computed as follows:

$$
\mathrm{LI}=\frac{(L-R)}{(L+C+R)},
$$

where $L$ and $R$ indicate the number of first fixations directed to the left and the right visual hemifield, respectively, and $C$ indicates the number of nonlateralized fixations.

\section{Statistical analysis}

Statistical analyses were carried out using PASW Statistics 18.0 (SPSS, Chicago, Illinois, USA) software. Group-level analyses were carried out using repeatedmeasure analysis of variance (ANOVA) with face category $(2 \times)$ as a within-participant factor and diagnostic group $(2 \times)$ as a between-participant factor. To further examine between-group differences within each face category, a one-way ANOVA was used. To test for a bias toward the left or the right visual hemifield, a one-sample $t$-test was used to compare the data against chance level (0) within each group separately. 

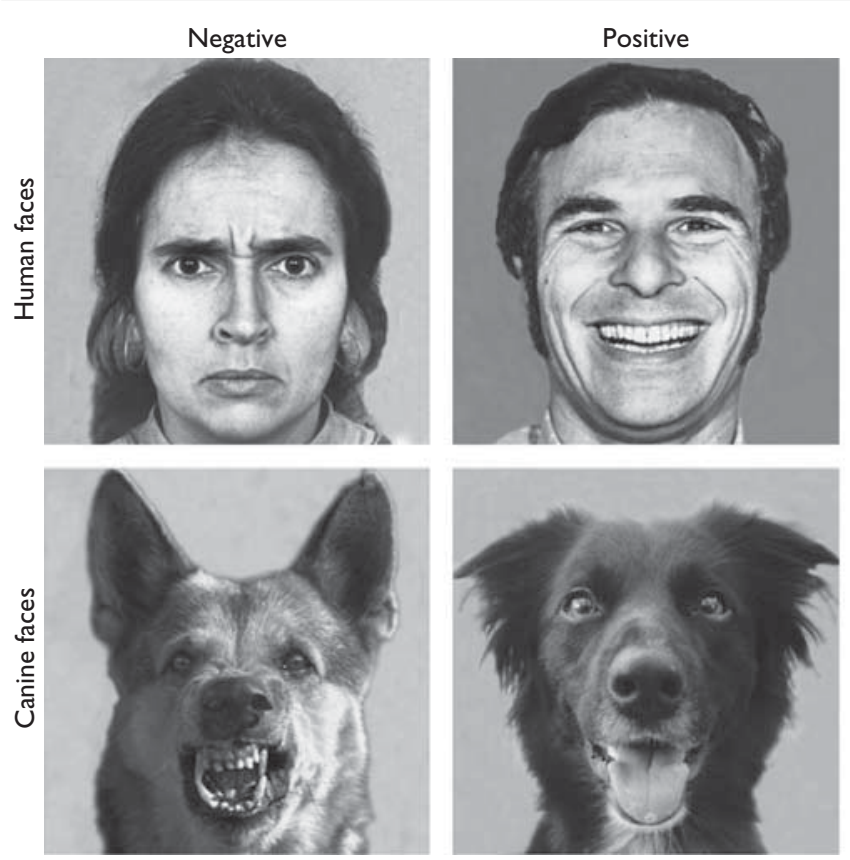
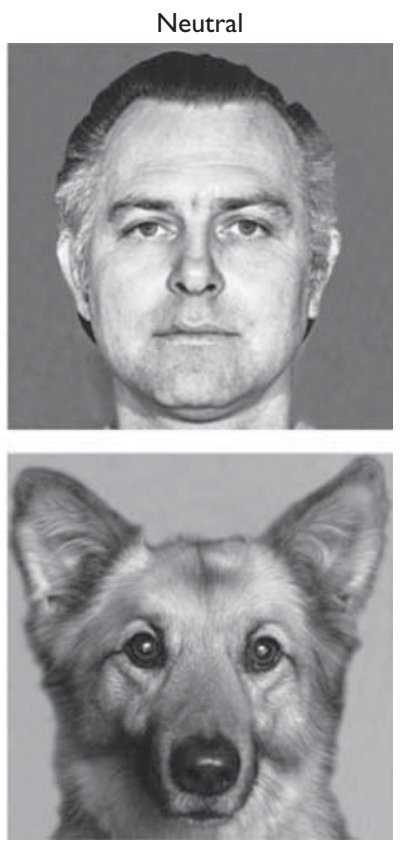

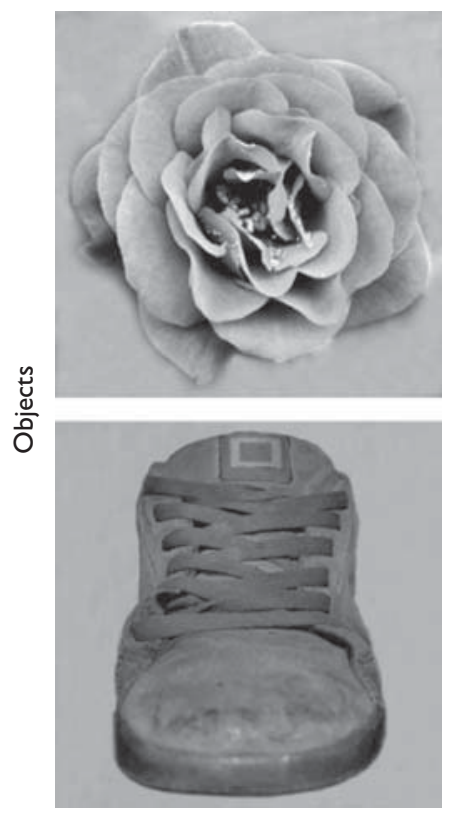

Example of stimuli.

Correlation analyses between laterality index and nonverbal and verbal mental age within each group did not show any association between these variables; thus, they were not entered as covariables in the between-group analyses.

\section{Results}

At least five valid trials per face category were needed to include the participant in the analyses. The number of valid trials for human faces was similar between groups $\left[\right.$ mean $_{\mathrm{ASD}}=9.1, \quad \mathrm{SD}=2.1 ; \quad \operatorname{mean}_{\mathrm{TD}}=9.6, \quad \mathrm{SD}=2.0$; $F(1,39)=0.86, P=0.360]$. For canine faces, the number of valid trials was higher for the TD group $\left[\operatorname{mean}_{\mathrm{ASD}}=9.8, \quad \mathrm{SD}=2.0 ; \quad\right.$ mean $_{\mathrm{TD}}=10.8, \quad \mathrm{SD}=1.4$; $F(1,39)=4.52, P=0.040]$.

The number of trials of symmetrical objects was less than 5 ; these were not included in the analysis.

\section{Emotional valence}

The Friedman test was used to examine the effect of emotional valence on the laterality index of the first fixation for each group and each category separately. Emotional valence had no effect on the laterality index of the first fixation for human faces $\left(\chi^{2}=1.53, P=0.465\right)$ and canine faces $\left(\chi^{2}=3.35, P=0.188\right)$ in ASD. Emotional valence for human faces did not have an effect in the TD group $\left(\chi^{2}=2.80, P=0.869\right)$. However, an effect of valence was observed on the laterality index for canine faces $\left(\chi^{2}=6.69, P=0.035\right)$. The Wilcoxon test between each emotional valence showed a difference between negative emotional valence and neutral emotional valence $(Z=-2.56, P=0.010)$.

\section{Laterality index}

An ANOVA with repeated category faces (human vs. canine) as the within-subject factor and group (ASD vs. TD) as the between-subject factor did not show a main effect of face category $[F(1,39)=0.01, P=0.924]$ and no interaction effect $[F(1,39)=1.19, \quad P=0.281]$. A main effect of group was found $[F(1,39)=4.12, P=0.049$, $f=0.33$ ] indicating that irrespective of the face (human or canine), TD were more likely to direct their first fixation to the left visual hemifield than the ASD group.

\section{Human faces}

The results of the one-way ANOVA indicate that for human faces, the TD group of children was more likely to direct the first fixation to the left visual hemifield than children in the ASD group $[F(1,39)=4.88, P=0.033$, $f=0.35]$ (Fig. 2).

For the ASD group, no gaze bias toward the left visual hemifield was found $\left[\operatorname{mean}_{\mathrm{ASD}}=0.08, \quad \mathrm{SD}=0.59\right.$; $t(18)=0.57, P=0.577]$. In contrast, for the TD group, the results showed a bias toward the left visual hemifield $\left[\right.$ mean $_{\mathrm{TD}}=0.43, \quad \mathrm{SD}=0.42 ; \quad t(21)=4.73, \quad P<0.001 ;$ $d=1.01]$.

For the ASD group, the proportion of children who showed a left visual hemifield gaze bias (47\%) was not different from that of a binomial probability distribution 
Fig. 2

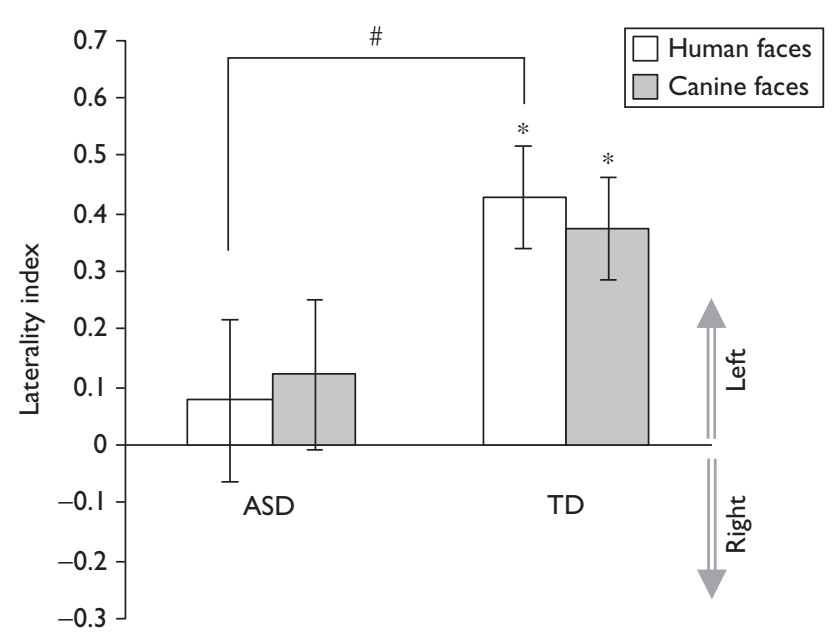

Mean laterality index and SE for the direction of the first fixation by group and by face category. ${ }^{\star} P<0.001$ (one-sample $t$-test), ${ }^{\#} P<0.05$ (oneway ANOVA).

of 0.5 (binomial test, $P=0.999$ ). In the TD group, a trend toward a significant proportion of children $(73 \%)$ having a bias for left lateralized first fixation was found (binomial test, $P=0.052$ ).

\section{Canine faces}

The results of the one-way ANOVA showed no significant difference between the ASD and the TD group of children for canine faces $[F(1,39)=2.79, P=0.103]$.

The one-sample $t$-test showed no gaze bias to the left visual hemifield for the ASD group $\left[\operatorname{mean}_{\mathrm{ASD}}=0.12, \mathrm{SD}=0.57\right.$; $t(18)=0.93, P=0.363]$. For the TD group, a gaze bias to the left visual hemifield was found $\left[\right.$ mean $_{\mathrm{TD}}=0.38, \mathrm{SD}=0.41$; $t(21)=4.34 ; P<0.001 ; d=0.93$ ] (Fig. 2).

For the ASD group, the proportion of children who showed a left visual hemifield gaze bias (58\%) was not different from that of a binomial probability distribution of 0.5 (binomial test, $P=0.648$ ). However, in the TD group, a significant proportion of children $(82 \%)$ had a bias for left lateralized first fixation (binomial test, $P=0.004, g=0.32)$.

\section{Discussion}

We confirmed our hypothesis of a lack of bias for the direction of the first fixation toward the left visual hemifield in children for ASD during the perception of human and canine faces, as well as a presence of this left bias for both face species in TD children.

These results in TD children are consistent with previous reports in young children $[3,7,20]$. They confirm that this bias appears early in development and that it is not specific to human faces. No effect of emotional valence was observed for the human face, replicating earlier results from Racca et al. [7]. However, for canine faces, an effect of valence was observed between negative and neutral faces. This is because of the fact that for canine faces with negative valence, more fixations were directed toward the mouth (i.e. not so lateralized) because it likely appears more prominent (teeth, tongue), which has the effect of reducing the laterality index in this study. This interpretation is consistent with the direction of the difference as the laterality index for faces with negative valence is lower than that of neutral faces (a gaze bias for the left visual hemifield is still observed irrespective of emotional valence).

A lack of bias for the left visual hemifield for the direction of the first fixation to human faces was observed in the group of children with ASD, which extends findings from Dundas et al. [14], who reported a lack of this bias for the total fixation time. These results suggest that unlike in TD children, the left visual hemifield that projects directly to the right hemisphere is not more favored than the right visual hemifield in response to faces presented centrally in children with ASD. A lack of bias toward the left visual hemifield for the direction of the first fixation was also observed for canine faces in children with ASD. This suggests that the absence of the bias is not specific to human faces, but applies to all faces with the first-order configuration, and suggests an anomaly at an early stage of visual analysis of faces. The overall results of the study reinforce the hypothesis of an alteration of the right hemispheric dominance and generally atypical hemispheric lateralization for face processing in individuals with ASD [11,12].

The lack of right hemispheric dominance for face processing may reflect a more general disorder of cerebral specialization of social functions in ASD. Anomalies are indeed observed in other areas in individuals with ASD, including difficulties in the areas of language, emotional perception, and motor lateralization. Interestingly, normal hemispheric lateralization in response to letters, a non-social domain, has been observed in ASD [12].

This study has a few limitations. First, because of their young age, the manual and ocular dominances of children were not evaluated, although several studies have noted some differences between children with ASD and typical children [21,22]. However, neither hand nor eye dominances seem to affect the left visual hemifield bias [5]. Further studies are nevertheless warranted, both in typical development and in ASD, to completely rule out any confounding effects of these factors. Second, because of their small number of trials, symmetrical objects could not be analyzed separately in this study. This could be the subject of future studies as it has been shown that the bias for the left visual hemifield for the direction of the first fixation can also be observed with stimuli other than faces [23] (but see Leonards and Scott-Samuel [5]). 
Future studies will be required to test whether the lack of this bias for face processing is specific to ASD or whether it is also present in other neurodevelopmental disorders. In addition, we believe that it would be worthwhile to test for the left visual hemifield gaze bias in first-degree relatives to further investigate whether a failure of normal right hemispheric dominance could reflect an endophenotypic marker of atypical face processing [20,24].

\section{Conclusion}

This study shows that young children with ASD failed to show a left visual hemifield bias for the direction of the first fixation for human and canine faces, likely reflecting a failure of normal right hemispheric dominance for face processing.

\section{Acknowledgements}

The authors are very grateful to the children and families who participated in this study. The authors would like to thank Dr Kun Guo for sharing the stimuli with us, as well as M.H. Afzali for his helpful advice.

This work was supported by a doctoral scholarship to QG from the Fondation Orange, by the ESF COST Action BM1004 Enhancing the scientific study of Early Autism (ESSEA) and by the Rossi Foundation and a Chaire d'Excellence Pierre de Fermat to N.H.

\section{Conflicts of interest}

There are no conflicts of interest.

\section{References}

1 Butler SH, Gilchrist ID, Burt DM, Perrett DI, Jones E, Harvey M. Are the perceptual biases found in chimeric face processing reflected in eyemovement patterns? Neuropsychologia 2005; 43:52-59.

2 Everdell IT, Marsh H, Yurick MD, Munhall KG, Paré M. Gaze behaviour in audiovisual speech perception: asymmetrical distribution of face-directed fixations. Perception 2007; 36:1535-1545.

3 Guo K, Meints K, Hall C, Hall S, Mills D. Left gaze bias in humans, rhesus monkeys and domestic dogs. Anim Cogn 2009; 12:409-418.

4 Guo K, Smith C, Powell K, Nicholls K. Consistent left gaze bias in processing different facial cues. Psychol Res 2012; 76:263-269.

5 Leonards U, Scott-Samuel NE. Idiosyncratic initiation of saccadic face exploration in humans. Vision Res 2005; 45:2677-2684.
6 Liu S, Quinn PC, Wheeler A, Xiao N, Ge L, Lee K. Similarity and difference in the processing of same- and other-race faces as revealed by eye tracking in 4- to 9-month-olds. J Exp Child Psychol 2011; 108:180-189.

7 Racca A, Guo K, Meints K, Mills D. Reading faces: differential lateral gaze bias in processing canine and human facial expressions in dogs and 4-yearold children. PLoS One 2012; 7:e36076.

8 Smith $\mathrm{Na}$, Gibilisco CR, Meisinger RE, Hankey M. Asymmetry in infants' selective attention to facial features during visual processing of infantdirected speech. Front Psychol 2013; 4:601.

9 Butler SH, Harvey M. Effects of aging and exposure duration on perceptual biases in chimeric face processing. Cortex 2008; 44:665-672.

10 CDC. Prevalence of autism spectrum disorder among children aged 8 years - autism and developmental disabilities monitoring network, 11 sites, United States, 2010. MMWR Surveill Summ 2014; 63:1-21.

11 McPartland J, Dawson G, Webb SJ, Panagiotides H, Carver LJ. Event-related brain potentials reveal anomalies in temporal processing of faces in autism spectrum disorder. J Child Psychol Psychiatry 2004; 45:1235-1245.

12 McPartland J, Wu J, Bailey Ca, Mayes LC, Schultz RT, Klin A. Atypical neural specialization for social percepts in autism spectrum disorder. Soc Neurosci $2011 ; 6: 436-451$.

13 Ashwin C, Wheelwright S, Baron-Cohen S. Laterality biases to chimeric faces in asperger syndrome: what is right about face-processing? J Autism Dev Disord 2005; 35:183-196.

14 Dundas E, Best Ca, Minshew N, Strauss MS. A lack of left visual field bias when individuals with autism process faces. J Autism Dev Disord 2012; 42:1104-1111.

15 Lord C, Risi S, Lambrecht L, Cook EH, Leventhal BL, DiLavore PC, et al. The autism diagnostic observation schedule-generic: a standard measure of social and communication deficits associated with the spectrum of autism. J Autism Dev Disord 2000; 30:205-223.

16 Lord C, Rutter M, Le Couteur A. Autism Diagnostic Interview-Revised: a revised version of a diagnostic interview for caregivers of individuals with possible pervasive developemental disorder. J Autism Dev Disord 1994; 24:659-685.

17 Mullen E. Mullen scales of early learning. Circle Pines: American Guidance Service; 1995.

18 Ekman P, Friesen W, Press C. Pictures of facial affect. CA: Palo Alto; 1975.

19 Wass S, Smith TJ, Johnson MH. Parsing eye-tracking data of variable quality to provide accurate fixation duration estimates in infants and adults. Behav Res Methods 2013; 45:229-250.

20 Dundas E, Gastgeb HZ, Strauss MS. Left visual field biases when infants process faces: a comparison of infants at high- and low-risk for autism spectrum disorder. J Autism Dev Disord 2012; 42:2659-2668.

21 Dane S, Balci N. Handedness, eyedness and nasal cycle in children with autism. Int J Dev Neurosci 2007; 25:223-226.

22 Hauck Ja, Dewey D. Hand preference and motor functioning in children with autism. J Autism Dev Disord 2001; 31:265-277.

23 Ossandón JP, Onat S, König P. Spatial biases in viewing behavior. J Vis 2014; 14:1-26.

24 Dawson G, Webb SJ, Wijsman E, Schellenberg G, Estes A, Munson J, et al. Neurocognitive and electrophysiological evidence of altered face processing in parents of children with autism: implications for a model of abnormal development of social brain circuitry in autism. Dev Psychopathol 2005; 17:679-697. 